\title{
High performance architecture design for large scale fibre-optic sensor arrays using distributed EDFAs and hybrid TDM/DWDM
}

\author{
Yi Liao ${ }^{a}$, Ed Austin ${ }^{b}$, Philip J. Nash ${ }^{b}$, Stuart A. Kingsley ${ }^{b}$, David J. Richardson ${ }^{a}$ \\ ${ }^{\mathrm{a} O p t o e l e c t r o n i c s ~ R e s e a r c h ~ C e n t r e, ~ U n i v e r s i t y ~ o f ~ S o u t h a m p t o n, ~ U K ; ~}$ \\ ${ }^{\mathrm{b}} \mathrm{TGS}$, Surrey KT6 6AP, UK \\ Email: yil@orc.soton.ac.uk
}

\begin{abstract}
A distributed amplified dense wavelength division multiplexing (DWDM) array architecture is presented for interferometric fibre optic sensor array systems. This architecture employs a distributed erbium doped fibre amplifier (EDFA) scheme to decrease the array insertion loss, and employs time division multiplexing (TDM) at each wavelength to increase the number of sensors that can be supported. The first experimental demonstration of this system is reported including results which show the potential for multiplexing and interrogating up to 4096 sensors using a single telemetry fibre pair with good system performance. The number can be increased to 8192 by using dual pump sources.
\end{abstract}

Keywords: Distributed amplified array, fibre sensor array, acoustic sensing, EDFA, multiplexing, phase resolution, noise aliasing

\section{INTRODUCTION}

Interferometric fiber optic acoustic sensors based on measuring the phase modulation of light travelling in an optical fiber due to the strains developed on the fibre by a measurand have been researched for nearly four decades. This research has been driven by their use in a number of important practical applications, for example underwater military sonar (hydrophones) and ocean bottom seismic sensing for oil and gas reservoir monitoring. Even with the ultra-high sensitivities available, single channel applications are only appropriate in a few instances for cost reasons. Fortunately, fibre-optic sensors lend themselves to multiplexing, which allows the cost of the lasers and other expensive components to be divided between many channels. Many multiplexing schemes have been proposed and investigated based on techniques including time, frequency, coherence, and 
wavelength multiplexing, and combinations thereof $[7,1-4]$. In each instance splitting/recombination loss ultimately limits the scalability of the approach in terms of the total number of sensors that can be accommodated, with the number of fibres required for telemetry a further critical factor that significantly impacts the overall system cost and practicality. The largest interferometric fibre-optic sensor array reported to date consisted of a time- and wavelength-division multiplexed architecture combining up to 256 sensors onto a single fibre pair [9].

In this paper, to our knowledge, we report the first experimental demonstration of acoustic measurements in an amplified TDM/DWDM array architecture. The system is capable of interrogating up to 4096 sensors through only one interlink fibre pair whilst satisfying the demanding performance levels required by many applications.

\section{PRINCIPLE OF OPERATION}

The principle of the array topology proposed here is based on loss compensation of the distribution bus using a distributed multi-section EDFA in a hybrid TDM/DWDM architecture. The array is addressed via two fibre buses, the distribution bus (top) and the return bus (bottom), as illustrated in Figure 1. The optical pulses from the wavelength multiplexed sources are coupled into the distribution bus. At the first optical drop multiplexer (ODM), the signal at wavelength $\lambda_{1}$ is coupled into the first TDM group (all sensors driven by one wavelength), passes through the constituent sub-array of TDM sensors, and is then coupled onto the return bus through the optical add multiplexer (OAM), which delivers it to the detector. The remaining wavelengths of the input interrogation signal continue along the distribution bus to the subsequent ODMs, successively feeding into each TDM group, before being multiplexed onto the return bus. This architecture performs the 'add' and 'drop' functions by separate devices, and thus provides the advantage that if the telemetry fibre is severed within the array then only the signals from the TDM groups corresponding to ODM/OAMs positioned after the break are lost. EDFA segments are distributed along the return bus to compensate the insertion loss, and the gain of each amplifying section is set to exactly compensate for the subsequent losses for each sensor group. The amplifiers are all co-pumped remotely through the existing return bus fibre by $1480 \mathrm{~nm}$ pump lasers located at the front and/or back ends of the array. The couplers are ODM/OAMs so that the pump never couples out of the bus. Since each amplifier must compensate for only a small insertion loss, (except for $\mathrm{amp}_{0}$ ), they provide a low gain and require just a small pump power. The input pump power is selected to be much larger than the first amplifier's saturation pump power. Therefore, this amplifier absorbs only a small fraction of the pump power so that a large remaining power is transmitted to the downstream amplifiers, which also operate in a pump saturation regime. 


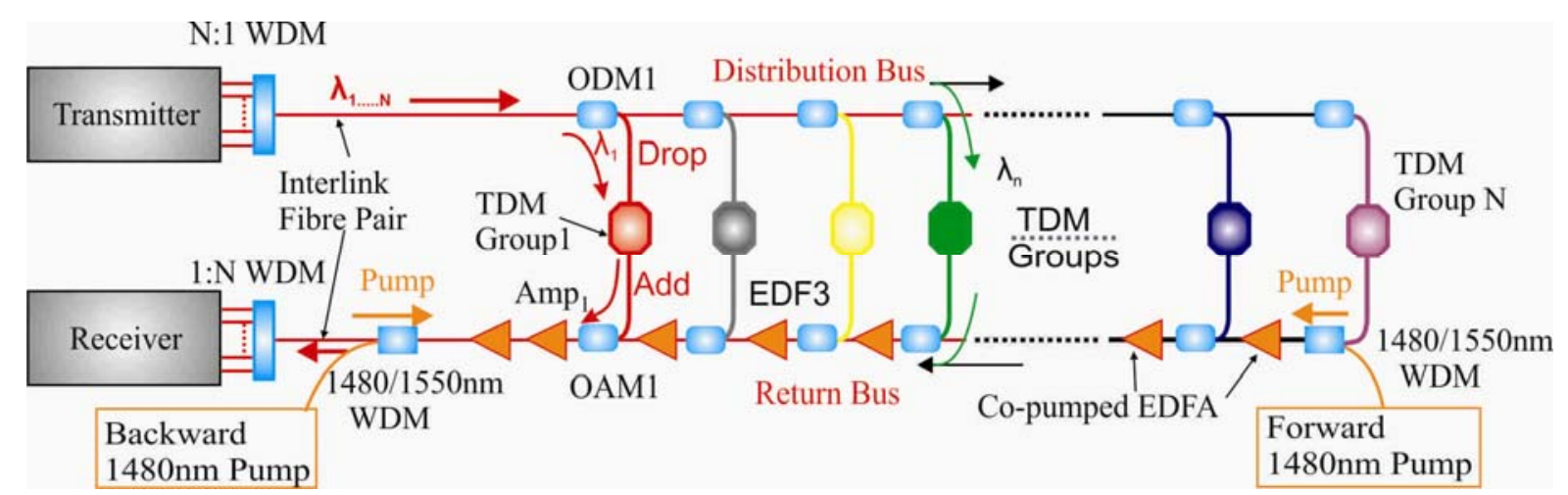

Figure 1 Proposed amplified TDM /DWDM array topology.

This array architecture provides dramatic advantages for large scale arrays. The topology uses TDM within amplified DWDM architectures for the first time, such that the number of multiplexed sensors is now given by the product of the number of TDM sensors and the number of wavelengths, which is increased by several times compared to the number multiplexed in amplified TDM arrays with the same interrogation repetition rate [3]. The interrogation repetition rate determines the bandwidth available for the phase modulated signal to occupy, and it is dependent on the number of sensors and the length of fibre per sensor. The insertion loss of the array is compensated by the distributed amplification, which leaves a higher power budget for the remote transmission of the signal along the cable, and limits the chance of nonlinear effects within the fibre bus. These benefits are of prime importance in many applications.

The array allows the interrogation of thousands of multiplexed sensors utilizing only one single telemetry fibre pair, which significantly decreases the array complexity, cost and weight, and provides for ready expansion. In particular, fewer telemetry fibres significantly reduce the complexity, weight and cost of the umbilical cabling between optical sensors. Fewer optical telemetry fibres also increase the possibility of using optical connectors. For instance the maximum number of optical fibres that can be joined with wet-mateable connectors undersea is currently 8 [5].

\section{EXPERIMENTAL SETUP}

Although a larger number of wavelengths might be multiplexed, due to cost, practicality and equipment availability, we use 16 wavelengths in our proof-of-principle experimental configuration, as shown in Figure 2. This sensor system comprises three principal components: a transmitter, the amplified array architecture and a receiver section. The transmitter comprises four narrow linewidth ( $\sim 10 \mathrm{kHz}$ ) fibre lasers for interrogation, and a further twelve DFB lasers (from a DWDM telecom source bank) to provide suitable signals to assess the optical amplification performance of the array (the linewidth of the DFB lasers was too broad to allow them to be used for sensor interrogation). The four narrow linewidth sources were multiplexed, pulsed, frequency-shifted and amplified, then launched into a "TDM group". The output signal was then combined with the twelve CW sources and 
launched into the amplified array architecture. The maximum launch peak power into the array was $+22 \mathrm{dBm}$ per wavelength, limited by nonlinear effects.

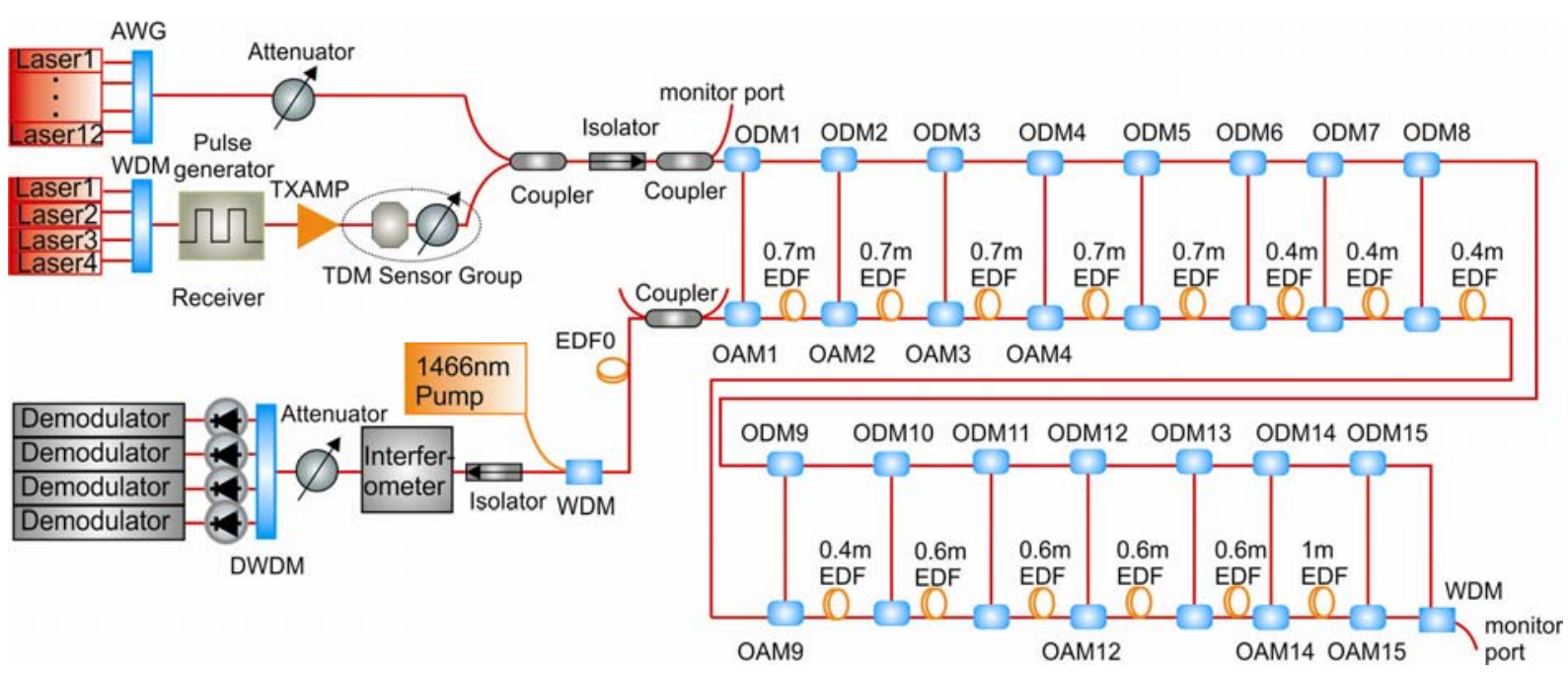

Figure 2 Example experimental setup of the 16 wavelength architecture for phase noise interrogation from 4 narrow linewidth fibre lasers and representing 12 DFB lasers. AWG: Arrayed waveguide gratings (SFT1000 from FIBERPRO), WDM: wavelength division multiplexer (P/N: DWDM108D33-CP1FC/APC from AC Photonics Inc. and the 1480/1550nm WDM from Fiberer, $54 \mathrm{~mm}$ ( $3 \mathrm{~mm}$ in size). ODM: Optical drop multiplexer; OAM: optical add multiplexer (3 Port DWDM from Fiberer, 40 $\mathrm{mm}$ ( $3 \mathrm{~mm}$ in size). EDF: Erbium doped fibre (I4 from Fibrecore).

The experimental arrangement was constructed with only one "TDM group" representing all of the sensors in the array. This was located before the array so that each wavelength suffered the loss of this device. This should provide similar optical performance in terms of loss to a fully-loaded system in which a TDM group is included between each ODM/OAM pair but is obviously far more convenient from a practical perspective. The "TDM group" itself comprised a single fibre Michelson interferometer sensor along with a tunable attenuator to simulate a prescribed number of additional "missing sensors" as described below. In the Michelson interferometric sensor, the input light is split into two fibre arms through a 50:50 fibre coupler, thus generating a signal beam and a reference beam. To simulate an applied acoustic signal, the fibre carrying the signal beam was wrapped around a piezoelectric transducer (PZT) thereby allowing the phase of light travelling through the fibre to be modulated (by applying an AC voltage to the PZT). Conversely, the reference beam is shielded from this phase perturbation. The signal and reference beams are then retroreflected using Faraday mirrors back towards the coupler where they recombine and interfere. The resulting beam on the up-lead is then fed into a demodulator, where the phase modulation along the signal arm is determined. The Michelson interferometers can be easily expanded to inline Michelson TDM architectures with only one coupler per sensor [6]. The sensors are time-multiplexed on a single line, and are separated by reflectors which are constructed by silvering one port of a fibre x-coupler. 
The amplified array comprises 15 stages of ODM/OAM-EDF along two fibre buses to support the 16 wavelengths. The insertion losses of the ODM/OAMs were measured to determine the lengths of Erdoped fibre required in each stage to provide sufficient gain to compensate for the insertion loss of the ODM/OAM pair. Since each amplifier stage compensates for only a small insertion loss, a lightlydoped fibre is chosen for our experiment, with Giles parameters as shown in Figure 3. The doped fibre has a relatively low peak absorption of $5 \mathrm{~dB} / \mathrm{m}$ at $1530 \mathrm{~nm}$. In our experiment all amplifiers were copumped by a single $1466 \mathrm{~nm}$ pump source coupled into the return bus. The maximum output pump power from the diode was $400 \mathrm{~mW}$. The lengths of amplifier segments used throughout the network were determined by exhaustive numerical simulations of the system, supplemented by a sequence of detailed amplifier measurements designed to allow us to determine the salient physical properties of the erbium doped fibre used.

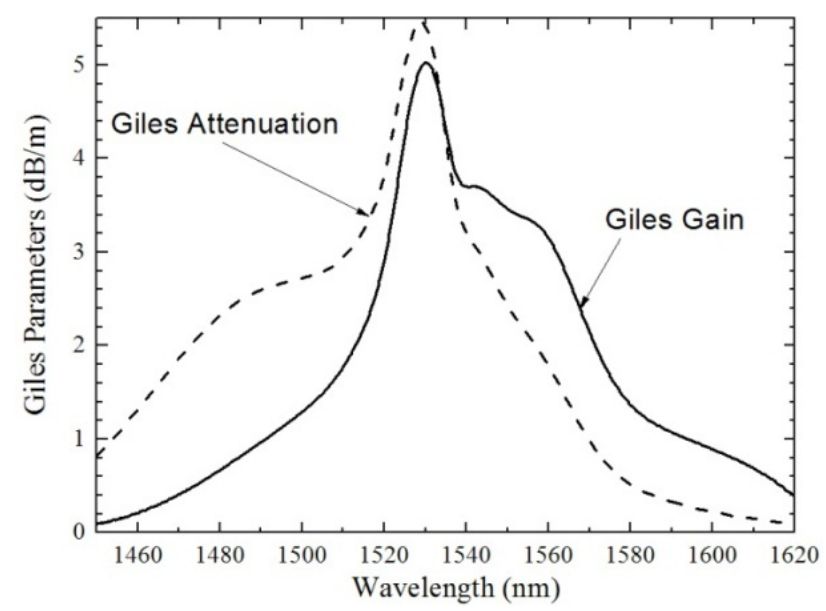

Figure 3 Giles parameters of the Er-doped fiber I4 used in the experimental setup.

The transmission spectra of the ODM/OAMs were also characterized to allow us to predict the optical performance of the amplified array, as illustrated in Figure 4. The figure shows a stop (pass) bandwidth of $0.4 \mathrm{~nm}$ on the pass (drop) channel, with a channel isolation of 20 (45) $\mathrm{dB}$, respectively. Although the non-ideal isolation of $20 \mathrm{~dB}$ results in a small amount of power being transmitted along the distribution bus and coupled back to the return bus through the last TDM group, this light undergoes a minimum optical suppression of $40 \mathrm{~dB}$ for a double 'pass' process through the multiplexers on both the distribution and return buses. Consequently, the crosstalk by this mechanism is $-80 \mathrm{~dB}$, and thus, this architecture will not introduce any additional sensor-to-sensor crosstalk between different TDM groups. Figure 5 shows the measured output amplified spontaneous emission (ASE) spectrum at the output of the amplified array in the absence of signal channel, which demonstrates a 'stop band' effect on the accumulated ASE noise. Since only signals with wavelengths out of the add channel bandwidth $(\sim 0.4 \mathrm{~nm})$ are passed through the pass channel of the OAMs on the return bus, and there exists a measured stop band attenuation, the OAMs also provide a partial rejection effect on the accumulated in-band ASE noise, shown as 'hollow' shapes around the multiplexer's center wavelength in the ASE spectrum. 


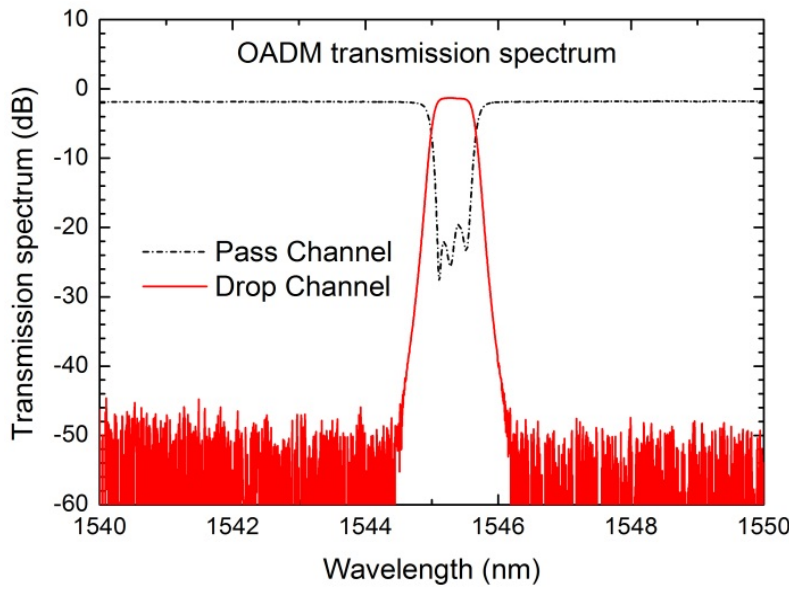

Figure 4 Optical transmission spectra at the pass channel and drop channel of the OADMs used in the experiment setup.

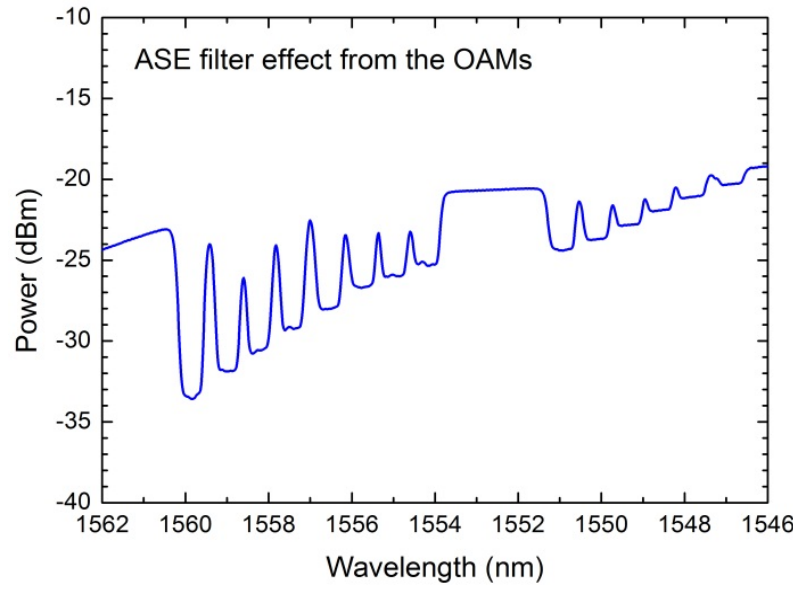

Figure 5 Demonstration of the filter effects on the accumulated ASE noise in the optical spectrum at the array output.

The return signals from the array were referenced at another, static reference interferometer for subtraction of system generated noise, and attenuated before the demultiplexer to achieve a peak optical power of $-27 \mathrm{dBm}$ per wavelength at the receiver as required to obtain shot noise limited performance. The demultiplexer was used to route the signal bearing channels to the correct receiver. The output of the demultiplexer was then detected and demodulated to extract the phase information.

\section{EXPERIMENTAL RESULTS}

The optical spectrum at the output of the array was characterized for a TDM group IL of $32 \mathrm{~dB}$, as illustrated in Figure 6. It can be seen from the spectrum that the optical signal to noise ratio (OSNR) for each channel is better than $35 \mathrm{~dB}$, which implies that the noise added by the distributed EDFA is sufficiently low that it can effectively be ignored [8].

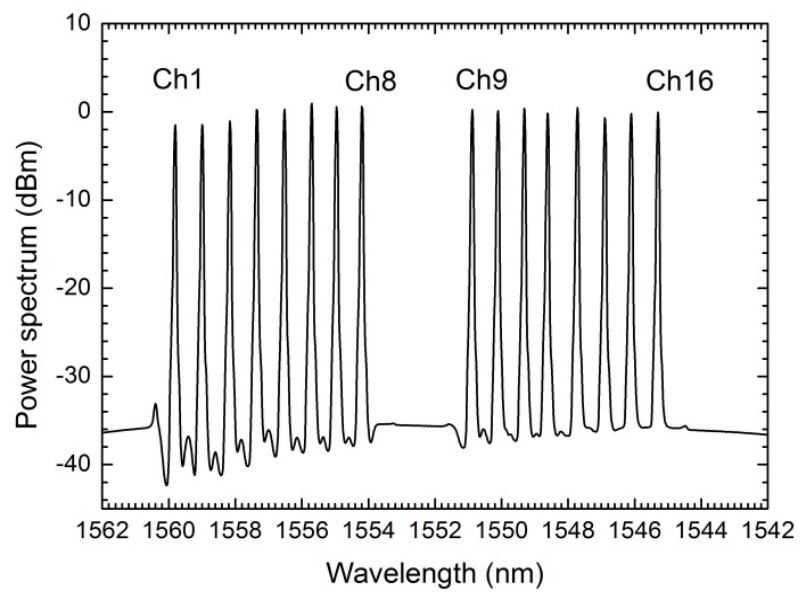

Figure 6 Optical spectrum from the array with the TDM group insertion loss equivalent to 256 sensors. The OSNR is larger than $35 \mathrm{~dB}$ at each channel.

To measure the system phase sensitivity, a sine-wave signal at $1 \mathrm{kHz}$ with an amplitude equivalent to a phase change of $-63 \mathrm{~dB}$ re $1 \mathrm{rad}$ in radians was applied via the PZT within one of the Michelson 
sensors in the "TDM group". The phase spectrum was tested over a wide range (29 to $59 \mathrm{~dB}$ ) of TDM group optical insertion loss (IL). The IL of one sensor was assumed to be $5 \mathrm{~dB}$, increasing by $6 \mathrm{~dB}$ for every doubling of the number of sensors in the TDM group, as is consistent with current sensor technology. The value of $6 \mathrm{~dB}$ originates from the insertion loss experienced by the returning light due to the additional 50:50 coupler required. Figure 7 shows the demodulated phase spectra range from $100 \mathrm{~Hz}$ to $2 \mathrm{kHz}$ interrogated at the last channel (1545.32 nm) with two TDM group ILs of 32 $\mathrm{dB}$ and $43 \mathrm{~dB}$, with the zoomed modulation-peak in the figure at the bottom. The IL for the two illustrated spectra in the figure is close to the loss of a TDM group with 16 and 64 sensors addressed by a single wavelength respectively. This equates to a total number of 256 and 1024 sensors respectively over 16 wavelengths. It can be seen from the figure that the system phase noise floor remains almost the same with increasing effective number of sensors ranging from 16 to 64 per wavelength, allowing a phase resolution of around $-90 \mathrm{~dB}$ re $1 \mathrm{rad} / \mathrm{Hz}^{1 / 2}$. The system phase noise floor shows a flat spectrum from 100 to $2000 \mathrm{~Hz}$ and thus we define the phase resolution in the sensor system as the mean value of the system phase noise floor from 300 to $800 \mathrm{~Hz}$, and the best system resolution is calibrated to be $-90 \mathrm{~dB}$ re $1 \mathrm{rad} / \mathrm{Hz}^{1 / 2}$ in a passive system limited only by the laser noise (below $100 \mathrm{~Hz}$ the noise is limited by environmental noise).

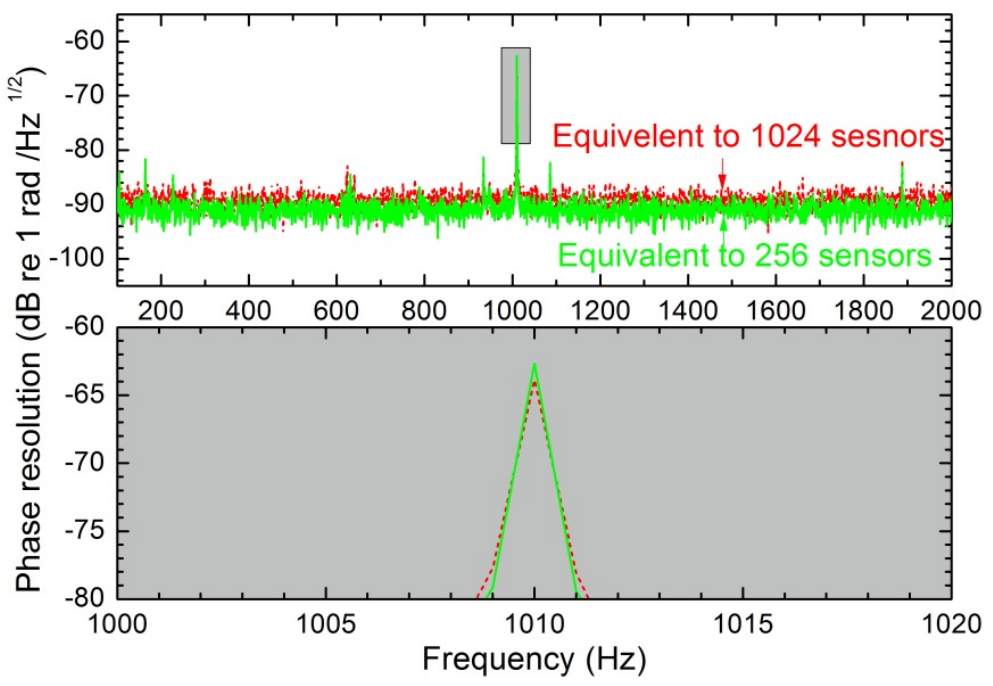

Figure 7 Demodulated phase spectra of the array output with TDM group insertion losses equivalent to 256 and 1024 sensors with 16 wavelengths. The peak corresponds to the ac modulation of the Michelson sensor.

\section{DISCUSSION}

The total number of sensors in the design can be further increased by increasing the number of sensors in each TDM group and the number of wavelength channels. However, the minimum phase information which can be detected is limited by the system phase noise floor which is determined by various factors including the receiver noise, shot noise, laser frequency noise, laser intensity noise, RF signal generator noise, ASE noise and noise aliasing in the TDM architecture. A detailed phase noise 
model has been developed for our system that takes into account all of these contributions and is presented in Reference [8]. Thus, the number of sensors in each TDM group cannot be increased indefinitely, due to two reasons. Firstly, because of the amplifier stages employed in the array, the signal to ASE beat noise contributes to the system noise floor. In the small-signal regime, due to the high population inversion, a low noise figure is obtained and the generated ASE noise can be ignored. However, as the IL of the TDM group is increased, a higher gain is required and a lower input signal is injected at the EDFA input. Consequently, ASE builds up rapidly and starts to saturate the EDFA giving rise to signal-ASE beat noise which ultimately becomes the dominant noise source, and which increases the system noise floor as the number of sensors per wavelength is increased.

The OSNR of the optical spectrum at the array output decreases with the IL of the TDM sensor group. Figure 8 demonstrates the two output optical spectra for different ILs of 43 and $53 \mathrm{~dB}$ in the TDM group. It is clear that following the increase of the IL of the TDM group, the ASE noise increases, and the output signal decreases; this results in a worse OSNR at the output optical spectra. It can also be seen from the figure that the 'stop band' effect from the ODMs improves the OSNR at the first channel by $10 \mathrm{~dB}$.

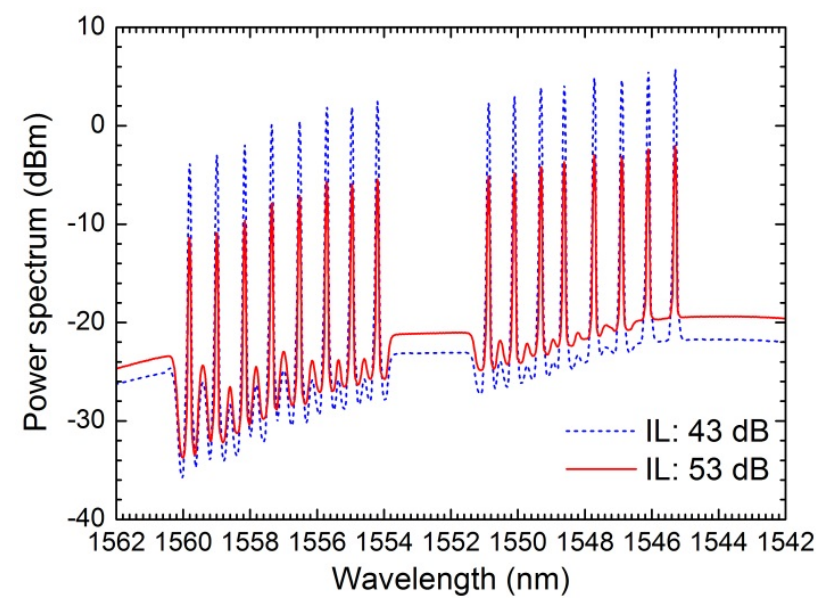

Figure 8 Optical spectra at the output of the array with different IL of the TDM group.

On the other hand, for a given pulse-width, the phase noise observed on each sensor increases with an increasing number of sensors in the TDM group, because the bandwidth available for each sensor decreases. TDM architectures inherently sample each sensor at the interrogation repetition rate which depends on the number of TDM sensors $M$ and the length of fibre per sensor $L_{s}$. The demodulation system samples the signal at the pulse repetition rate with $f_{s}=f_{\mathrm{rep}}$. If the high frequency noise is up to the electrical bandwidth of the receiver $E_{*} \gg \frac{f_{z}}{2}$, the Nyquist sampling condition is not satisfied. Adjacent copies overlap, and any frequency component above $\frac{f_{z}}{2}$ is indistinguishable from a lowerfrequency component, called an alias, as illustrated in Figure 9. When the high frequency noise is 
white, which is the case for the signal to ASE beat noise in a limited bandwidth of $B_{\theta}$, the effective aliased bandwidth in the experimental setup is given by

$$
B_{a}=2 B_{e} / f_{\text {rep }} \propto B_{e} L_{s}(M+1)
$$

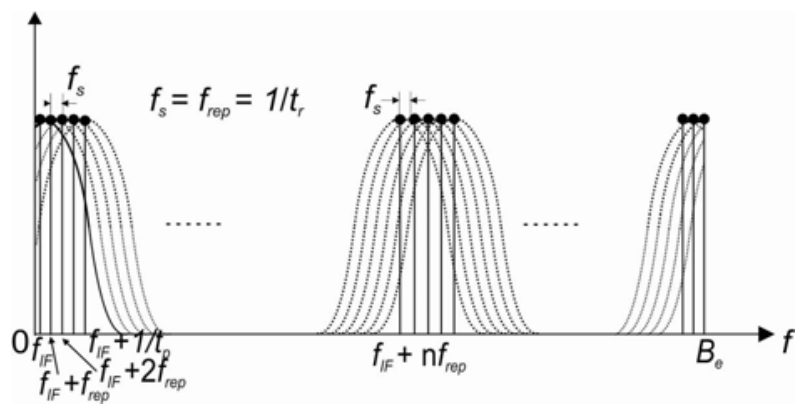

Figure 9 Noise aliasing in the demodulation system.

Thus, the phase noise floor at the low frequencies of interest increases with the number of sensors in each TDM group. The table below demonstrates the relationship between the number of sensors addressed by a single wavelength, the total number of sensors with 16 wavelength channels, the aliased bandwidth, and the predicted phase resolution with an electrical bandwidth of $120 \mathrm{MHz}$. The predicted phase resolution covers both the increasing ASE noise induced phase noise and the aliased high frequency noise. It can be seen from the table that the expected noise floor is increased to -77 $\mathrm{dB}$ re $1 \mathrm{rad} / \mathrm{Hz}^{1 / 2}$ when the number of sensors is increased to 256 per wavelength.

Table 1 Predicted phase resolution as a function of number of sensors with specified pulse width and sampling rate

\begin{tabular}{ccccc}
\hline $\begin{array}{c}\text { Number of } \\
\text { sensors }\end{array}$ & $\begin{array}{c}\text { Total number } \\
\text { of sensors }\end{array}$ & $\begin{array}{c}\text { Sampling rate } \\
(\mathrm{Hz})\end{array}$ & $\begin{array}{c}B_{a} \\
(\mathrm{~Hz})\end{array}$ & $\begin{array}{c}\text { Phase resolution } \\
\left(\mathrm{dB} \mathrm{re} 1 \mathrm{rad} / \mathrm{Hz}^{1 / 2}\right)\end{array}$ \\
\hline 1 & 16 & $200 \mathrm{k}$ & 1200 & -91 \\
16 & 256 & $200 \mathrm{k}$ & 1200 & -90 \\
64 & 1024 & $200 \mathrm{k}$ & 1200 & -88 \\
128 & 2048 & $100 \mathrm{k}$ & 2400 & -82 \\
256 & 4096 & $50 \mathrm{k}$ & 4800 & -77 \\
\hline
\end{tabular}

The maximum number of wavelengths in the array is limited by the insertion loss of the return bus at the pump wavelength and the available maximum output power of the pump sources. The transmission loss of the pump power is dominated by the insertion loss of the ODMs. The pump power decreases rapidly along the return bus, thus we can increase the number of wavelength channels in the array by constructing a dual-pump source configuration as presented in Figure 1. 


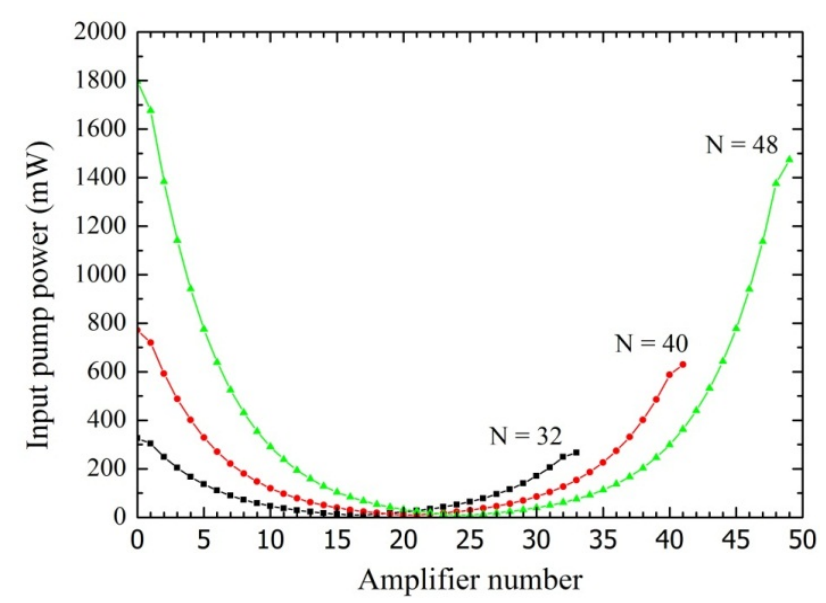

Figure 10 Pump power incident at each amplifier stage for an array with 32, 40, and 48 amplifier stages in the return bus with dual-pump sources. Parameters values are the signal peak power $P_{\boldsymbol{s}}$ of $+22 \mathrm{dBm}$ per channel, input pump power selected such that $10 \mathrm{~mW}$ is incident on the middle amplifier for dual pumped architecture.

The required pump power increases with an increase in the number of EDF-ODM/OAMs (or the number of wavelengths). Figure 10 shows the required pump power for an array with 32, 40, and 48 amplifier stages (equivalent to the number of wavelengths) along the return bus with pump sources at both ends. The required forward and backward pump pairs for 32, 40 and 48 wavelengths are 325/267 $\mathrm{mW}, 771 / 630 \mathrm{~mW}$, and 1800/1473 $\mathrm{mW}$, respectively. With dual pump sources of 2 ( $400 \mathrm{~mW}$, we can support at least 32 channels of wavelengths in the architectural design presented, thus the total number of sensors can be increased to $256 \times 32$ with an acceptable phase noise floor of $-77 \mathrm{~dB}$ re $1 \mathrm{rad} / \mathrm{Hz}^{1 / 2}$.

To summarize, our experimental results predict that our system can address up to $64 \times 16$ sensors with a phase resolution limited only by the noise of our lasers. Further consideration on the maximum number of sensors shows that the design can support up to $256 \times 32$ by using an amplifier system with dual pumps with a perfectly acceptable phase resolution for many applications of $-77 \mathrm{~dB}$ re 1 $\mathrm{rad} / \mathrm{Hz}^{1 / 2}$, even with an adjusted sampling rate.

\section{CONCLUSION}

In conclusion, we have demonstrated what is believed to be the first experiment addressing acoustic sensing in a distributed amplified array based on a hybrid TDM and DWDM architecture. Our experimental results show that the array is able to support a total number of 1024 sensors along one interlink fibre pair, allowing a phase resolution of around $-90 \mathrm{~dB}$ re $1 \mathrm{rad} / \mathrm{Hz}^{1 / 2}$, limited only by laser noise. Further, we show that the current 16-wavelength array could theoretically be expanded to interrogate 4096 sensors, albeit with a slight compromise in phase resolution. The maximum number of interrogation sensors could be further increased to 8192 by using dual pump sources. We consider that this architecture represents the best approach to support a distributed interferometric sensing array 
of more than 1000 sensors through one pair of interlink fibres, using only commercially available components.

\section{REFERENCES}

[1] Cranch G A and Nash P J 2001 Large-scale multiplexing of interferometric fiber-optic sensors using TDM and DWDM Journal of Lightwave Technology 19 687-99

[2] Geoffrey A C and Phillip J N 1999 High multiplexing gain using TDM and WDM in interferometric sensor arrays. In: SPIE Conference on Fiber Optic Sensor Technology and Applications, pp 531-7

[3] Hodgson C W, Digonnet M J and Shaw H J 1997 Large-scale interferometric fiber sensor arrays with multiple optical amplifiers Opt Lett 22 1651-3

[4] Hodgson C W, Wagener J L, Digonnet M J F and Shaw H J 1998 Optimization of large-scale fiber sensor arrays incorporating multiple optical amplifiers. Journal of Lightwave Technology 16 218-31

[5] Jenkins D and Christiansen M 2012 Enabling technology: High capacity wet mateable optic Connection. In: Oceans, 2012: IEEE) pp 1-7

[6] Kirkendall C K and Cole J H 2006 Progress in fiber optic acoustic and seismic sensing Optical Society of America Technical Digest

[7] Kirkendall C K and Dandridge A 2004 Overview of high performance fibre-optic sensing Journal of Physics D: Applied Physics 37 R197

[8] Liao Y, Austin E, Nash P J, Kingsley S A and Richardson D J 2013 Phase resolution characterization in fiber-optic sensor systems using amplifiers and TDM. (Submitted to: Journal of Lightwave Technology)

[9] Nash P J and Strudley A 2009 High efficiency TDM/WDM architectures for seismic reservior monitoring. In: 20th International Conference on Optical Fiber Sensors, pp T1-T4 